\title{
Gesture Performance in First- and Multiple-Episode Patients with Schizophrenia Spectrum Disorders
}

\author{
Katharina Stegmayer ${ }^{\mathrm{a}}$ Jeanne Moor ${ }^{\mathrm{a}}$ Tim Vanbellingen ${ }^{\mathrm{b}, \mathrm{c}}$ \\ Stephan Bohlhalter ${ }^{c}$ René M. Müri ${ }^{b}$ Werner Strik $^{\mathrm{a}}$ Sebastian Walther ${ }^{\mathrm{a}}$ \\ a University Hospital of Psychiatry, and ${ }^{b}$ Department of Neurology and Clinical Research, Perception and \\ Eye Movement Laboratory, University Hospital, Inselspital, Bern, and ' $N e u r o l o g y$ and Neurorehabilitation Center, \\ Lucerne Cantonal Hospital, Lucerne, Switzerland
}

\author{
Key Words \\ Action planning $\cdot$ Hand gesture $\cdot$ Imitation . \\ Neurodevelopment $\cdot$ Nonverbal communication . \\ Pantomime
}

\begin{abstract}
Background/Aim: Gesturing plays an important role in social behavior and social learning. Deficits are frequent in schizophrenia and may contribute to impaired social functioning. Information about deficits during the course of the disease and presence of severity and patterns of impairment in first-episode patients is missing. Hence, we aimed to investigate gesturing in first- compared to multiple-episode schizophrenia patients and healthy controls. Methods: In 14 first-episode patients, 14 multiple-episode patients and 16 healthy controls matched for age, gender and education, gesturing was assessed by the comprehensive Test of Upper Limb Apraxia. Performance in two domains of gesturing imitation and pantomime - was recorded on video. Raters of gesture performance were blinded. Results: Patients with multiple episodes had severe gestural deficits. For almost all gesture categories, performance was worse in multiple- than in first-episode patients. First-episode patients demonstrated subtle deficits with a comparable pattern. Conclusions:
\end{abstract}

Subjects with multiple psychotic episodes have severe deficits in gesturing, while only mild impairments were found in first-episode patients independent of age, gender, education and negative symptoms. The results indicate that gesturing is impaired at the onset of disease and likely to further deteriorate during its course.

(c) 2016 S. Karger AG, Basel

\section{Introduction}

Social impairment is a cardinal feature of schizophrenia and present throughout the course of illness starting at the prodromal stage [1]. Nonverbal communication is critical for successful social interaction [2]. Patients with schizophrenia suffer from severe problems in nonverbal information processing. They have difficulties in perceiving and recognizing nonverbal social cues [3]. This has been repeatedly shown for emotion recognition of facial expression [4] and is also true for other nonverbal cues, such as visual body cues (hands and body posture) as well as vocal nonverbal cues $[5,6]$. Furthermore, patients display nonverbal actual face-to-face interaction deficits [7]. These deficits have been associated with negative symptoms [8] and reduced social competence [9].

\section{KARGER}

E-Mail karger@karger.com

www.karger.com/nps
(C) 2016 S. Karger AG, Basel

0302-282X/16/0734-0201\$39.50/0
Katharina Stegmayer

University Hospital of Psychiatry Bern

Bolligenstrasse 111

CH-3060 Bern (Switzerland)

E-Mail stegmayer@puk.unibe.ch 
Table 1. Clinical characteristics of first-episode patients $(n=14)$, multiple-episode patients $(n=14)$ and healthy controls $(n=16)$

\begin{tabular}{lllllrl}
\hline & First episode & Multiple episodes & Healthy controls & d.f. & F/T/ $\chi^{2}$ & $\mathrm{p}$ \\
\hline Age, years & $27.2 \pm 8.5$ & $28.1 \pm 4.6$ & $27.7 \pm 6.3$ & 2 & 0.4 & 0.690 \\
Males, \% & 78.6 & 78.6 & 56.3 & 2 & 2.4 & 0.296 \\
Education & $14.4 \pm 3.0$ & $14.3 \pm 3.0$ & $14.8 \pm 2.8$ & 2 & 0.1 & 0.886 \\
PANSS pos. & $17.1 \pm 6.8$ & $14.5 \pm 5.1$ & - & 1 & 1.1 & 0.267 \\
PANSS neg. & $17.7 \pm 5.7$ & $18.1 \pm 5.0$ & - & 1 & -0.2 & 0.862 \\
PANSS gen. & $35.2 \pm 10.9$ & $33.9 \pm 7.6$ & - & 1 & 0.4 & 0.719 \\
PANSS total & $70.0 \pm 19.3$ & $66.5 \pm 14.2$ & - & 1 & 0.5 & 0.589 \\
CPZ & $225.9 \pm 170.8$ & $414.5 \pm 336.9$ & - & 1 & -1.9 & 0.073 \\
FAB & $16.8 \pm 1.5$ & $16.9 \pm 1.5$ & $17.8 \pm 0.4$ & 2 & 2.9 & 0.072 \\
MRS & $1.4 \pm 1.7$ & $3.4 \pm 3.5$ & - & 1 & -2.0 & 0.058 \\
AIMS glob. & $0.1 \pm 0.4$ & $1.1 \pm 1.5$ & - & 1 & -2.3 & 0.027 \\
UPDRS-3 & $2.6 \pm 3.3$ & $5.6 \pm 6.1$ & - & 1 & -1.6 & 0.116 \\
MMSE & $29.3 \pm 1.0$ & $27.9 \pm 2.5$ & - & 1 & 2.0 & 0.061 \\
\hline
\end{tabular}

$\mathrm{CPZ}=\mathrm{CPZ}$-equivalent dosage; FAB = Frontal Assessment Battery; MRS = Modified Rogers Scale; glob. = global judgment.

One critical feature in nonverbal communication is gesturing $[10,11]$. Gestures can for instance enhance language understanding [12], and mediate social learning and memory processes $[13,14]$. Recently, gesture performance has been shown to be impaired in patients with schizophrenia. Simple imitation of meaningless manual and oral gestures was impaired [9]. In addition, it was shown that pantomiming meaningless gestures was particularly disturbed [15], which is associated with poor frontal lobe function [16]. Furthermore, disturbed nonverbal social perception is linked to poor gesture performance in schizophrenia [17].

Schizophrenia often encompasses multiple episodes with progressive deterioration. Some psychosis-related neuropsychological deficits occur early in the course of the disease. Patients may present with deficits in memory, and executive and motor functioning after remission of the first episode [18]. Neurocognitive deficits such as verbal executive and verbal memory deficits, for example, are found early on, with a clear trend toward decline during the course of the disorder [19]. Likewise, motor abnormalities in patients with schizophrenia were present before treatment. In fact, motor abnormalities have also been observed in high-risk samples $[20,21]$ in children who later developed schizophrenia [22] and in unmedicated first-episode patients with psychosis $[23,24$, for review see 25]. Interestingly, some motor deficits in firstepisode patients exacerbated acutely during antipsychotic treatment (i.e. over the first few months) and then gradually returned to baseline levels with continued treatment $[26,27]$. Furthermore, subjects at risk for psychosis demonstrate reduced and faulty gesturing $[28,29]$.

The aim of the current study was to establish whether gestural deficits are present at the first psychotic episode. Furthermore, the study explored whether gestural deficits were likely to progress during the course of the illness, contrasting patients with first and multiple episodes. Patients were matched for age, gender and education. We expected to find gestural impairments in first-episode patients with lower severity and frequency than in patients with multiple episodes.

\section{Participants and Methods}

\section{Participants}

Twenty-eight inpatients (14 first-episode patients and 14 multiple-episode patients) of the University Hospital of Psychiatry, Bern (Switzerland), meeting the diagnostic criteria of schizophrenia, schizoaffective disorder or schizophreniform disorder according to DSM-IV, and 16 healthy control subjects participated in this study. Diagnoses were given after thorough clinical examination and review of all case files by board-certified psychiatrists and were ascertained by the Structured Clinical Interview for DSM (SCID). All study participants provided written informed consent. The study protocol adhered to the Declaration of Helsinki and was approved by the local ethics committee. General exclusion criteria were a history of traumatic brain injury, or concurrent alcohol or substance dependence. All subjects were right handed, which was assessed with the Edinburgh Handedness Inventory [30]. Age, gender and duration of education did not differ between groups (table 1). 
Table 2. Gesture performance of first- and multiple-episode patients and healthy controls

\begin{tabular}{llll}
\hline TULIA & First episode & Multiple episodes & Healthy controls \\
\hline Total left & $220.6 \pm 14.2(21)$ & $205.6 \pm 17.9(57)$ & $228.2 \pm 5.4(0)$ \\
Total right & $222.3 \pm 12.9(21)$ & $210.9 \pm 18.3(21)$ & $227.3 \pm 7.2(0)$ \\
Imitation left & $112.7 \pm 5.4(7)$ & $103.7 \pm 10.9(21)$ & $114.4 \pm 2.4(0)$ \\
Pantomime left & $107.9 \pm 9.3(21)$ & $101.9 \pm 10.5(50)$ & $113.8 \pm 4.2(0)$ \\
Imitation right & $112.3 \pm 6.1(14)$ & $106.7 \pm 11.1(21)$ & $114.5 \pm 2.8(0)$ \\
Pantomime right & $110.0 \pm 8.3(21)$ & $104.1 \pm 9.2(36)$ & $112.8 \pm 5.1(0)$ \\
\hline
\end{tabular}

Means \pm SD. Percent deficits are shown in parentheses. Note that TULIA cutoff scores were determined for the left hand.

Healthy controls were volunteers recruited from the hospital staff and the community. They were screened using the Mini International Neuropsychiatric Interview [31] to exclude any concurrent or previous history of axis I psychiatric disorders. None of the controls took psychotropic medication or suffered from any neurological or major medical condition. Controls had no firstdegree relative with a psychotic disorder.

In the patient groups, dosages of antipsychotic medication were assessed and chlorpromazine (CPZ)-equivalent doses were calculated according to Woods [32]. CPZ equivalents did not differ between the patient groups (table 1) with 3 patients being off antipsychotic medication at the time of participation. Abnormal involuntary movements were more frequent in multiple-episode patients. The mean duration of illness in the multiple-episode patient group was $9.3 \pm 5.2$ years.

\section{Procedures}

Diagnoses were given following clinical interviews, review of all records available as well as SCID. Psychopathology was assessed by trained raters using the Positive and Negative Syndrome Scale (PANSS) [33]. In addition, frontal lobe function, motor behavior and broad cognitive function were assessed using the Frontal Assessment Battery [34], the Modified Rogers Scale [35], the Abnormal Involuntary Movement Scale (AIMS) [36], the Unified Parkinson's Disease Rating Scale motor part (UPDRS-3) [37] and the Mini-Mental State Examination (MMSE) [38].

\section{Gesture Tests}

Participants performed the Test of Upper Limb Apraxia (TULIA) [39] with the left and the right arm separately. Briefly, the TULIA assesses the performance of meaningless, transitive (toolrelated) and intransitive (symbolic non-tool-related) gestures in two domains, i.e. imitation (performance after demonstration) and pantomime (performance following verbal instruction). The order of tests in both arms and both presentation domains was randomized across the participants. The performance was videotaped and rated by an expert rater blinded to clinical information and diagnoses. Each of the 48 items is rated on a scale from 0 (no movement) to 5 (correct performance), taking spatial, temporal and content errors such as body part as an object into account. The test can be obtained at www.tulia.ch, and instructions are given in the original publication. Previously, cutoff scores were determined for each semantic category for the left arm in a group of younger adults [15].

Gesturing in First- and Multiple-Episode Schizophrenia Patients

\section{Statistical Analyses}

Statistical tests were performed using SPSS 21.0 (SPSS Inc., Chicago, Ill., USA). Two-sample t tests, one-way analysis of variance (ANOVA) and $\chi^{2}$ tests were used to test continuous and categorical clinical variables between patients and healthy controls.

Gesture performance for both arms was tested between the patient groups and healthy controls. First, we used a repeated-measure ANOVA with group as between-subject factor and hand (left and right), domain (imitation and pantomime) and semantic category (meaningless, intransitive and transitive gestures) as withinsubject factors. Greenhouse-Geisser correction was applied. Post hoc tests between the groups (multiple- vs. first-episode patients, multiple-episode patients vs. controls and first-episode patients vs. controls) were calculated and corrected for multiple comparisons (Sidak correction).

Next, to further disentangle triple interactions of repeatedmeasure ANOVA, we analyzed all semantic categories independent of the performance hand (mean left and right hand) using multivariate ANOVA (MANOVA) over all groups. Post hoc tests for MANOVA were corrected for multiple comparisons (Sidak correction).

In addition, exploratory analyses were conducted to test differences between first-episode patients and controls using nonparametric Mann-Whitney U tests. For these results, we calculated effect sizes using Rosenthal's $\mathrm{r}$ (small effect $>0.1$; medium effect $>$ 0.3 , and large effect $>0.5[40]$ ). Level of significance was set at $\mathrm{p}<$ 0.05 (two tailed).

Finally, we assessed the association of antipsychotic medication dosage (CPZ) with abnormal involuntary movements (AIMS scores) and gesture performance using Pearson's correlation in the patients.

\section{Results}

\section{Gesture Impairments in Schizophrenia}

Applying the cutoff scores determined previously for the left hand [15], 50\% of the multiple-episode and $21 \%$ of the first-episode patients presented deficits in pantomime gestures (gesture performance on verbal command), and $21 \%$ of the multiple-episode and $7 \%$ of the 
Table 3. Gesture performance of first- and multiple-episode patients and healthy controls: effects of hand, domain, category, group and interaction effects

\begin{tabular}{|c|c|c|c|c|c|c|c|c|c|c|c|c|}
\hline \multicolumn{12}{|c|}{ Repeated-measure ANOVA } & \multirow{3}{*}{$\begin{array}{l}\text { Post hoc test: effect of group } \\
\mathrm{p}\end{array}$} \\
\hline & \multicolumn{3}{|l|}{ effect } & & \multicolumn{3}{|l|}{ effect } & & \multicolumn{3}{|l|}{ effect } & \\
\hline & $\mathrm{F}$ & d.f. & $\mathrm{p}$ & & $\mathrm{F}$ & d.f. & $\mathrm{p}$ & & $\mathrm{F}$ & d.f. & $\mathrm{p}$ & \\
\hline Hand & 2.769 & 1.0 & 0.104 & hand $\times$ group & 2.299 & 2.0 & 0.113 & hand $\times$ domain $\times$ group & 1.289 & 2.0 & 0.286 & controls $>$ multiple episodes \\
\hline Domain & 5.922 & 1.0 & 0.019 & domain $\times$ group & 0.497 & 2.0 & 0.612 & domain $\times$ category $\times$ group & 3.207 & 3.4 & 0.017 & $<0.001$ \\
\hline Category & 36.500 & 1.9 & $<0.001$ & category $\times$ group & 3.944 & 3.9 & 0.006 & hand $\times$ domain $\times$ category & 0.292 & 2.0 & 0.747 & first $>$ multiple episodes \\
\hline \multirow[t]{3}{*}{ Group } & 8.981 & 2.0 & $<0.001$ & hand $\times$ domain & 0.028 & 1.0 & 0.898 & hand $\times$ domain $\times$ category $\times$ group & 0.879 & 3.5 & 0.480 & 0.026 \\
\hline & & & & hand $\times$ category & 1.409 & 1.7 & 0.250 & & & & & controls $>$ first episode \\
\hline & & & & domain $\times$ category & 3.207 & 2.0 & 0.017 & & & & & 0.462 \\
\hline
\end{tabular}

Table 4. Gesture performance of first- and multiple-episode patients and healthy controls in each semantic category

\begin{tabular}{|c|c|c|c|c|c|c|c|c|c|}
\hline & \multicolumn{6}{|c|}{ MANOVA (Wilks' $\lambda=0.469 ; \mathrm{F}=2.759 ;$ d.f. $=24 ; \mathrm{p}=0.004$ ) } & \multicolumn{3}{|c|}{ Post hoc test: Sidak correction } \\
\hline & $\begin{array}{l}\text { first } \\
\text { episode }\end{array}$ & $\begin{array}{l}\text { multiple } \\
\text { episodes }\end{array}$ & $\begin{array}{l}\text { healthy } \\
\text { controls }\end{array}$ & $\mathrm{F}$ & d.f. & $\mathrm{p}$ & $\begin{array}{l}\text { multiple episodes } \\
\text { vs. controls }\end{array}$ & $\begin{array}{l}\text { first vs. multiple } \\
\text { episodes }\end{array}$ & $\begin{array}{l}\text { controls vs. } \\
\text { first episode }\end{array}$ \\
\hline \multicolumn{10}{|c|}{ Mean TULIA: imitation } \\
\hline Meaningless & $37.2 \pm 2.3$ & $35.1 \pm 3.0$ & $38.8 \pm 1.3$ & 10.015 & 2 & $<0.001$ & $p<0.001$ & $\mathrm{p}=0.051$ & $\mathrm{p}=0.178$ \\
\hline Intransitive & $38.6 \pm 1.4$ & $36.8 \pm 4.2$ & $39.4 \pm 0.9$ & 4.135 & 2 & 0.023 & $p=0.022$ & $\mathrm{p}=0.169$ & $\mathrm{p}=0.801$ \\
\hline Transitive & $36.7 \pm 1.4$ & $33.4 \pm 5.6$ & $36.3 \pm 1.6$ & 3.799 & 2 & 0.031 & $p=0.017$ & $p=0.049$ & $\mathrm{p}=0.989$ \\
\hline \multicolumn{10}{|c|}{ Mean TULIA: pantomime } \\
\hline Meaningless & $34.8 \pm 4.6$ & $30.8 \pm 4.7$ & $37.4 \pm 2.3$ & 10.562 & 2 & $<0.001$ & $p<0.001$ & $p=0.032$ & $\mathrm{p}=0.209$ \\
\hline Intransitive & $38.6 \pm 2.0$ & $36.8 \pm 4.0$ & $39.0 \pm 1.2$ & 2.972 & 2 & 0.062 & $\mathrm{p}=0.075$ & $\mathrm{p}=0.198$ & $\mathrm{p}=0.971$ \\
\hline Transitive & $35.6 \pm 3.1$ & $36.4 \pm 3.0$ & $36.8 \pm 2.0$ & 1.257 & 2 & 0.295 & $\mathrm{p}=0.411$ & $\mathrm{p}=0.502$ & $\mathrm{p}=0.999$ \\
\hline
\end{tabular}

first-episode patients showed deficits in gesture imitation (performance after demonstration). None of the control subjects showed any deficit (table 2).

The repeated-measure ANOVA demonstrated significant effects of gesture domain, gesture category and group (table 3). In addition, we found a significant effect of the interactions category $\times$ group and of domain $\times$ category $x$ group. In contrast, no significant effect emerged for performance hand or any of the interactions with performance hand (table 3 ). The corrected post hoc tests demonstrated that multiple-episode patients experienced more problems performing gestures than healthy controls and first-episode patients (table 3).

In addition, we investigated gesture performance between the groups in each semantic category irrespective of the performing hand using MANOVA (table 4; fig. 1). Multiple-episode patients displayed more difficulties performing gestures in most categories than healthy controls, and more difficulties performing some categories than first-episode patients (table 4). No significant differ- ences in gesture performance were detected between healthy controls and first-episode patients.

In detail, the performance of meaningless gestures was more impaired in both patient groups and both gesture domains. Most pronounced differences in gesture performance between multiple-episode patients and healthy controls were detected in meaningless gestures in the imitation and pantomime domain. The group differences were less prominent for intransitive (symbolic) gestures. In contrast, transitive (tool-related) pantomime performance was unaffected. Patient groups (first vs. multiple episodes) differed mainly in pantomime performance of meaningless gestures, with more deficits in the chronic patients (table 4; fig. 1).

\section{Exploratory Analysis of Gesture Impairments in \\ First-Episode Patients}

Comparing first-episode patients and healthy controls, no significant differences in gesture performance were found using post hoc tests with correction for mul- 


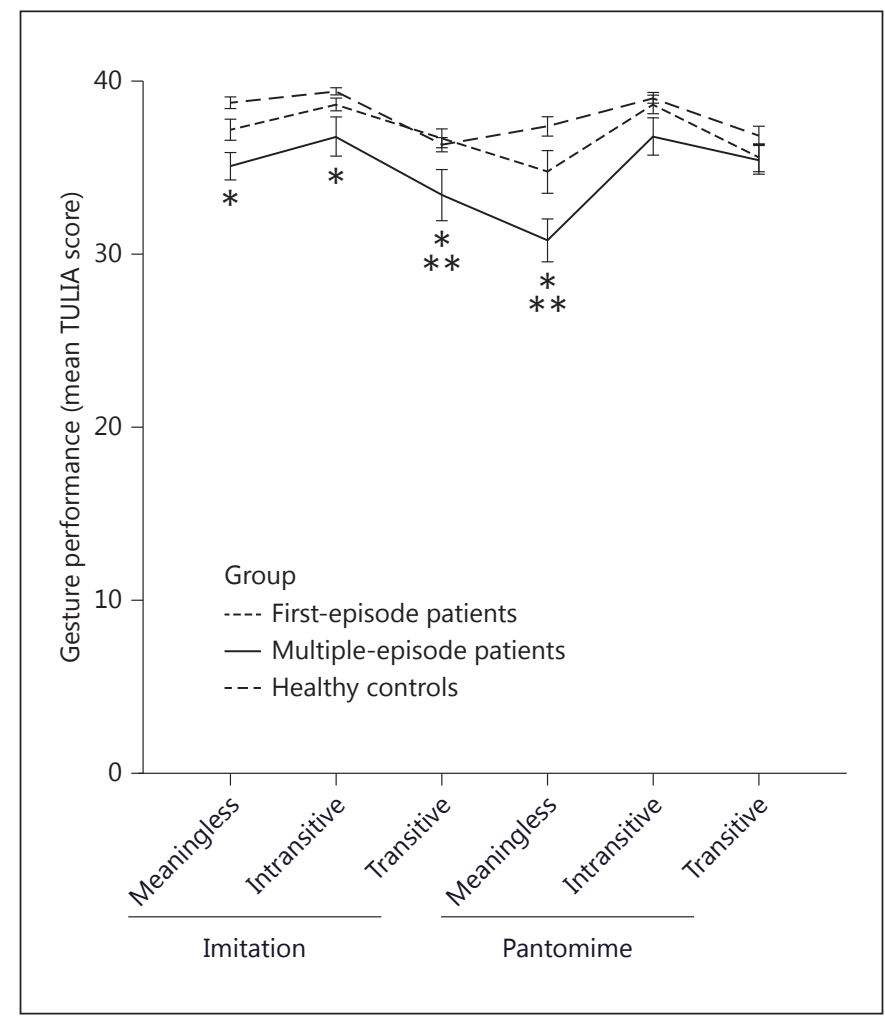

Fig. 1. Gesture performance of first- and multiple-episode patients and healthy controls in each semantic category. Lines indicate means \pm SEM. ${ }^{*} \mathrm{p}<0.05$, multiple-episode patients vs. healthy controls; ${ }^{* *} \mathrm{p}<0.05$, multiple- vs. first-episode patients.

tiple comparisons (tables 3, 4). However, some first-episode patients clearly presented severe performance deficits with scores below the cutoff scores (table 2 and online suppl. fig. A; for all online suppl. material, see www. karger.com/doi/10.1159/000446116). We therefore aimed to further explore whether first-episode patients would differ from controls in gesture performance applying exploratory analyses.

First-episode patients performed poorer than controls for both hands (right hand: $\mathrm{z}=-2.10 ; \mathrm{p}=0.036 ; \mathrm{r}=0.27$, and left hand: $\mathrm{z}=-2.65 ; \mathrm{p}=0.008 ; \mathrm{r}=0.31$ ). Exploring gesture domains (imitation and pantomime), performance of imitation of the left hand $(\mathrm{z}=-3.32 ; \mathrm{p}=0.001$; $r=3.4$ ) was particularly disturbed. The same was true for the right hand but to a lesser degree, with a trend for significance $(\mathrm{z}=-1.73 ; \mathrm{p}=0.084 ; \mathrm{r}=0.25)$. Moreover, pantomime of the right hand $(\mathrm{z}=-2.14 ; \mathrm{p}=0.032 ; \mathrm{r}=0.28)$ was impaired in first-episode patients. For additional exploratory analysis of the semantic subcategories, see online supplementary material (suppl. analysis A).

Gesturing in First- and Multiple-Episode Schizophrenia Patients
Noteworthy, all first-episode patients $(n=5)$ with gesture performance below the cutoff scores (TULIA total scores) showed a positive family history for psychosis, while this was the case only for 1 first-episode patient without gesture performance deficits below the cutoff scores. However, this patient scored only 2 points above the cutoff score for TULIA total, but below some cutoff scores of the gesture categories. Moreover, comparing the duration of the episode prior to the test did not differ between first-episode patients with and without gesture performance deficits [patients with deficits: duration of episodes $6-36$ weeks prior to the test $(16.1 \pm 8.7)$; patients without deficits $6-22$ weeks $(13.0 \pm 6.7) ; \mathrm{T}=-0.64 ; \mathrm{p}=$ 0.536].

Finally, in patients, no significant associations of antipsychotic medication dosage (CPZ) and AIMS scores with gesture performance (TULIA total scores) were shown (left hand: CPZ and TULIA total scores: $r=-0.243$; $\mathrm{p}=0.212$, AIMS global scores and TULIA total scores: $\mathrm{r}=-0.341 ; \mathrm{p}=0.076$; right hand: $\mathrm{CPZ}$ and TULIA total scores: $\mathrm{r}=-0.275 ; \mathrm{p}=0.157$; AIMS global scores and TULIA total scores: $\mathrm{r}=-0.335 ; \mathrm{p}=0.080$ ).

\section{Discussion}

The present study investigated gesture performance in first- and multiple-episode schizophrenia patients and healthy controls. We applied a comprehensive test of gesture performance including two domains: imitation (performance after demonstration) and pantomime (performance following verbal instruction) with blinded video ratings [39]. Patients with multiple episodes showed severe deficits in performing gestures with both hands. Performance deficits were particularly prominent during meaningless and intransitive gestures. Performance of first-episode patients was not significantly worse than performance of healthy controls. However, a proportion of first-episode patients (21\%) presented substantial gesture performance errors, e.g. body part as an object, omission and spatial orientation errors, leading to performance rates below the cutoff scores in some patients [15]. Exploratory analysis revealed subtle deficits in gesture performance with a comparable pattern of deficits in first- and multiple-episode patients. Particularly, meaningless gesture performance was affected.

We hypothesized that patients with first-episode schizophrenia would demonstrate gestural impairments. The findings of the present investigation partially support this notion. As noted, gesture performance was difficult 
in some but not all first-episode patients, and exploratory analyses revealed mild impairments in gesture performance compared to healthy controls. Particularly the performance of meaningless gestures remained difficult, as previously reported in schizophrenia patients [15]. These deficits have specifically been associated with impaired frontal lobe function [16]. In general, the frontal lobe is relevant for higher-order motor control, including planning and execution [41]. Indeed, schizophrenia patients with gesture deficits present reduced gray matter volume in the left inferior frontal gyrus [42]. Performance of meaningless (novel) gestures may be more demanding in terms of frontally mediated action planning than transitive and intransitive gestures, which are sought to be highly overlearned gestures [39]. We may, therefore, speculate that in patients with slightly disturbed action planning, performance of the more demanding gestures (meaningless gestures) is affected, while the performance of highly overlearned gestures (transitive and intransitive gestures) is still preserved. In real life, nonverbal communication relies frequently on familiar gestures. However, sometimes familiar gestures are not sufficient and we need to generate new gestures with changing context. Thus, defective action planning may impair performance of novel gestures, which in turn disturbs correct nonverbal communication.

Our findings are suggestive of an effect of familial load on gesture performance. Those who had clear-cut deficits among the first-episode patients also had a positive family history of psychosis. Future studies should, therefore, attempt to identify gesture deficits and a possible genetic vulnerability to the illness. In order to rule out effects of the disease process on gesture deficits among first-episode patients, we compared the duration of the episode prior to the test with no obvious difference between firstepisode patients with and without gesture performance deficits. In sum, a subgroup of first-episode patients presented with clear gestural deficits, and the exploratory analysis revealed a poorer performance compared with healthy subjects for the entire group in almost all gesture categories.

Our hypothesis that gestural impairments would be less prominent in first-episode patients than in patients with multiple episodes was confirmed. Patients with multiple episodes showed severer impairment in gesture performance (higher error rates) in almost all gesture categories. Thus, our findings are consistent with a progressive decline in gesture performance during the course of the disease rather than a stable deficit. In fact, first-episode patients demonstrated a similar pattern of difficulties in gesture impairments as the multiple-episode patients. Still, the frequency of errors was much higher in patients with multiple episodes than in first-episode patients. The differences in this cross-sectional analysis are unlikely to stem from effects of age, medication or negative symptoms. In fact, first- and multiple-episode patients did not differ in age, education, gender, PANSS scores or CPZ dosage in our study. Moreover, groups did not differ in the presentation of motor signs such as parkinsonism or catatonia. This supports the assumption that deterioration in gesture performance is likely to be a result of the process of the illness itself rather than driven by external effects. Still, AIMS scores differ between both patient groups. This is in line with the literature showing an increase in abnormal movements with increasing duration of illness [43]. This is also true for never-treated at-risk subjects [44]. Yet, AIMS scores as well as CPZ dosage did not correlate with gesture performance in patients in a previous report [16] and the current study.

In our study, the majority of first-episode patients showed largely preserved performance when producing gestures on command. In contrast, two reports of spontaneous gesture use suggest alterations in gesture use in unmedicated schizotypal adolescents and youth at ultrahigh risk for psychosis $[28,29]$. The authors investigated the spontaneous frequency of well-defined gesture categories, such as iconic, metaphoric, beat and deictic (pointing) gestures, during a natural interview situation. Besides the quantitative reduction in spontaneous gesture use [29], subjects at risk also demonstrated qualitative alterations in gesture content [28]. In fact, increased mismatch errors (incongruence between content of speech and gesture) and more retrieval gestures (gestures during speech pause while the participant is searching an expression) were reported in subjects at risk. These data suggest that gesture use is altered before the onset of frank psychosis. Our results support the notion that gesture performance is altered already in the early phase of the disorder and grossly deteriorated in chronic patients. The difference in the results between these studies is very likely due to the methods applied. TULIA focuses on a set of specific hand gestures that need to be replicated as precisely as possible. The task posits specific underlying demands for the gesture categories tested. Specific motor skills and matching action, for instance, are required during imitation. Therefore, imitation deficits possibly reflect severe motor, observation and matching action deficits. These neuropsychological deficits in gesture imitation in schizophrenia have been reported by others $[9,45]$. Furthermore, understanding of gestures in a specific context and 
underlying neuronal correlates was investigated in schizophrenia patients $[46,47]$. The authors noted specific difficulties in gesture understanding in an abstract sentence context (metaphoric gestures). They assume a dysfunctional integration of multimodal communication processing (speech and gesture - verbal and visual) in schizophrenia patients. With the current study, we did not test gesture perception or actual gesture use. It is, however, conceivable that impaired gesture performance would be related to poor gesture perception in patients with schizophrenia spectrum disorders.

In summary, our findings confirm that gesture performance is frequently impaired in schizophrenia patients and suggest, together with previous findings, that at least slight impairment is present early in the course of the disorder, prompting the question about their pathophysiological and clinical significance. In the nonverbal domain, gesture performance deficits may contribute to poor communicative functioning. It has been demonstrated that in the absence of gestures, language is difficult to understand [10]. Furthermore, deficits in nonverbal communication contribute to poor social functioning of patients with schizophrenia [2]. In the light of these findings, it is likely that inefficient gesture performance may contribute to impaired social functioning and functional outcome. Given that poor social perception impaired gesture performance [17], poor gesture performance in firstepisode patients may result from poor social cognition at the very onset of the disorder. In fact, deficits in social cognition were reported even before the actual onset of psychosis, for instance in adolescents at risk for psychosis [48], and may already substantially impact social functioning in first-episode patients.
The present study has some limitations. The sample size was relatively small. In addition, most patients had been exposed to antipsychotics prior to study participation; therefore, deficits could be partly attributed to both the disorder itself and to the effects of antipsychotic treatment. In particular, multiple-episode patients have been exposed to long-term antipsychotic treatment, which was not the case in first-episode patients. Still, groups did not differ in terms of age, gender, education and extrapyramidal motor function. Finally, this was a cross-sectional study, and longitudinal analyses of gesture performance are clearly needed.

In conclusion, we found that multiple psychotic episodes were associated with severe deficits in gesture performance compared to the first episode independent of age, gender, education and negative symptoms. Firstepisode patients showed relatively preserved gesture performance (although some deficits were detected) even if they are not entirely devoid of gesture performance impairment. Therefore, our results indicate that gesture performance deficits occur early and are likely to continuously decline during the course of the disease.

\section{Acknowledgments}

This work was supported by the Swiss National Science Foundation (SNSF: 152619/1 to S.W. and S.B.) and the BangerterRhyner Foundation (to S.W.).

\section{Disclosure Statement}

The authors declare that they have no conflict of interest.

\section{References}

1 Cornblatt BA, Auther AM, Niendam T, Smith CW, Zinberg J, Bearden CE, et al: Preliminary findings for two new measures of social and role functioning in the prodromal phase of schizophrenia. Schizophr Bull 2007;33:688702.

2 Couture SM, Penn DL, Roberts DL: The functional significance of social cognition in schizophrenia: a review. Schizophr Bull 2006; 32(suppl 1):S44-S63.

3 Penn DL, Sanna LJ, Roberts DL: Social cognition in schizophrenia: an overview. Schizophr Bull 2008;34:408-411.

4 Kalkstein S, Hurford I, Gur RC: Neurocognition in schizophrenia. Curr Top Behav Neurosci 2010;4:373-390.

Gesturing in First- and Multiple-Episode Schizophrenia Patients
5 Borod JC, Welkowitz J, Alpert M, Brozgold AZ, Martin C, Peselow E, et al: Parameters of emotional processing in neuropsychiatric disorders: conceptual issues and a battery of tests. J Commun Disord 1990;23:247-271.

-6 Toomey R, Schuldberg D, Corrigan P, Green MF: Nonverbal social perception and symptomatology in schizophrenia. Schizophr Res 2002;53:83-91.

7 Lavelle M, Healey PG, McCabe R: Nonverbal behavior during face-to-face social interaction in schizophrenia: a review. J Nerv Ment Dis 2014;202:47-54.

-8 Demirbuga S, Sahin E, Ozver I, Aliustaoglu S, Kandemir E, Varkal MD, et al: Facial emotion recognition in patients with violent schizophrenia. Schizophr Res 2013;144:142-145.
\$9 Park S, Matthews N, Gibson C: Imitation, simulation, and schizophrenia. Schizophr Bull 2008;34:698-707.

10 Beattie G, Shovelton H: Iconic hand gestures and the predictability of words in context in spontaneous speech. Br J Psychol 2000;91: 473-491.

11 Walther S, Mittal VA: Why we should take a closer look at gestures. Schizoph Bull 2016; 42:259-261.

12 Obermeier C, Dolk T, Gunter TC: The benefit of gestures during communication: evidence from hearing and hearing-impaired individuals. Cortex 2012;48:857-870. 
13 Straube B, Meyer L, Green A, Kircher T: Semantic relation vs surprise: the differential effects of related and unrelated co-verbal gestures on neural encoding and subsequent recognition. Brain Res 2014;1567:42-56.

14 Heyes C: Causes and consequences of imitation. Trends Cogn Sci 2001;5:253-261.

15 Walther S, Vanbellingen T, Müri R, Strik W, Bohlhalter S: Impaired gesture performance in schizophrenia: particular vulnerability of meaningless pantomimes. Neuropsychologia 2013;51:2674-2678.

16 Walther S, Vanbellingen T, Müri R, Strik W, Bohlhalter S: Impaired pantomime in schizophrenia: association with frontal lobe function. Cortex 2013;49:520-527.

-17 Walther S, Stegmayer K, Sulzbacher J, Vanbellingen T, Müri R, Strik W, et al: Nonverbal social communication and gesture control in schizophrenia. Schizophr Bull 2015;41:338345.

18 Bilder RM, Goldman RS, Robinson D, Reiter G, Bell L, Bates JA, et al: Neuropsychology of first-episode schizophrenia: initial characterization and clinical correlates. Am J Psychiatry 2000;157:549-559.

19 Pukrop R, Schultze-Lutter F, Ruhrmann S, Brockhaus-Dumke A, Tendolkar I, Bechdolf A, et al: Neurocognitive functioning in subjects at risk for a first episode of psychosis compared with first- and multiple-episode schizophrenia. J Clin Exp Neuropsychol 2006; 28:1388-1407.

20 Marcus J, Hans SL, Lewow E, Wilkinson L, Burack CM: Neurological findings in highrisk children: childhood assessment and 5-year followup. Schizophr Bull 1985;11:85100.

21 Mittal VA, Tessner KD, Trottman HD, Esterberg M, Dhruv SH, Simeonova DI, et al: Movement abnormalities and the progression of prodromal symptomatology in adolescents at risk for psychotic disorders. J Abnorm Psychol 2007;116:260-267.

22 Walker E, Lewine RJ: Prediction of adult-onset schizophrenia from childhood home movies of the patients. Am J Psychiatry 1990;147: 1052-1056.

23 Peralta V, Cuesta MJ: Neuromotor abnormalities in neuroleptic-naive psychotic patients: antecedents, clinical correlates, and prediction of treatment response. Compr Psychiatry 2011;52:139-145.
Peralta V, Campos MS, De Jalon EG, Cuesta MJ: Motor behavior abnormalities in drugnaive patients with schizophrenia spectrum disorders. Mov Disord 2010;25:1068-1076.

25 Walther S, Strik W: Motor symptoms and schizophrenia. Neuropsychobiology 2012;66: 77-92.

26 Bilder RM, Bates JA: Neuropsychological prediction of treatment response and outcome in schizophrenia; in Gaebel W, Awad AG (eds): Prediction of Neuroleptic Treatment Outcome in Schizophrenia. Vienna, Springer, 1994, pp 99-110.

27 Bachmann S, Bottmer C, Schröder J: Neurological soft signs in first-episode schizophrenia: a follow-up study. Am J Psychiatry 2005; 162:2337-2343

28 Millman ZBG, J, Schiffman J, Mejias J, Gupta T, Mittal VA: Mismatch and lexical retrieval gestures are associated with visual information processing, verbal production, and symptomatology in youth at high risk for psychosis. Schizophr Res 2014;158:64-68.

29 Mittal VA, Tessner KD, McMillan AL, Delawalla Z, Trotman HD, Walker EF: Gesture behavior in unmedicated schizotypal adolescents. J Abnorm Psychol 2006;115:351-358.

30 Oldfield RC: The assessment and analysis of handedness: the Edinburgh Inventory. Neuropsychologia 1971;9:97-113.

31 Sheehan DV, Lecrubier Y, Sheehan KH, Amorim P, Janavs J, Weiller E, et al: The Mini-International Neuropsychiatric Interview (M.I.N.I.): the development and validation of a structured diagnostic psychiatric interview for DSM-IV and ICD-10. J Clin Psychiatry 1998;59(suppl 20):22-33; quiz 34-57.

32 Woods SW: Chlorpromazine equivalent doses for the newer atypical antipsychotics. J Clin Psychiatry 2003;64:663-667.

33 Kay SR, Fiszbein A, Opler LA: The Positive and Negative Syndrome Scale (PANSS) for Schizophrenia. Schizophr Bull 1987;13:261276.

34 Dubois B, Slachevsky A, Litvan I, Pillon B: The FAB: a Frontal Assessment Battery at bedside. Neurology 2000;55:1621-1626.

-35 Lund CE, Mortimer AM, Rogers D, McKenna PJ: Motor, volitional and behavioural disorders in schizophrenia. 1. Assessment using the Modified Rogers Scale. Br J Psychiatry 1991;158:323-327, 333-336.

36 Guy W: ECDEU Assessment Manual for Psychopharmacology, revised ed. Washington, US Department of Health, Education, and Welfare, 1976.
37 Fahn SE, Elton RL: Unified Parkinson's disease rating scale; in Fahn S, Marsden CD, Calne DB, Goldstein M (eds): Recent Developments in Parkinson's Disease. Florham Park, Macmillan Healthcare Information, 1987.

38 Folstein MF, Folstein SE, McHugh PR: 'Minimental state'. A practical method for grading the cognitive state of patients for the clinician. J Psychiatr Res 1975;12:189-198.

39 Vanbellingen T, Kersten B, Van Hemelrijk B, Van de Winckel A, Bertschi M, Müri R, et al: Comprehensive assessment of gesture production: a new Test of Upper Limb Apraxia (TULIA). Eur J Neurol 2010;17:59-66.

40 Cohen J: Statistical Power Analysis for the Behavioral Science, ed 2. Hillsdale, Erlbaum, 1988.

41 Miller EK, Cohen JD: An integrative theory of prefrontal cortex function. Annu Rev Neurosci 2001;24:167-202.

42 Stegmayer K, Bohlhalter S, Vanbellingen T, Federspiel A, Moor J, Wiest R, et al: Structural brain correlates of defective gesture performance in schizophrenia. Cortex 2016;78: 125-137.

43 Quinn J, Meagher D, Murphy P, Kinsella A, Mullaney J, Waddington JL: Vulnerability to involuntary movements over a lifetime trajectory of schizophrenia approaches $100 \%$, in association with executive (frontal) dysfunction. Schizophr Res 2001;49:79-87.

44 Mittal VA, Neumann C, Saczawa M, Walker EF: Longitudinal progression of movement abnormalities in relation to psychotic symptoms in adolescents at high risk of schizophrenia. Arch Gen Psychiatry 2008;65:165-171.

45 Matthews N, Gold BJ, Sekuler R, Park S: Gesture imitation in schizophrenia. Schizophr Bull 2013;39:94-101.

46 Straube B, Green A, Sass K, Kircher T: Superior temporal sulcus disconnectivity during processing of metaphoric gestures in schizophrenia. Schizophr Bull 2014;40:936-944.

47 Straube B, Green A, Sass K, Kirner-Veselinovic A, Kircher T: Neural integration of speech and gesture in schizophrenia: evidence for differential processing of metaphoric gestures. Hum Brain Mapp 2013;34:1696-1712.

48 Dworkin RH, Cornblatt BA, Friedmann R, Kaplansky LM, Lewis JA, Rinaldi A, et al: Childhood precursors of affective vs social deficits in adolescents at risk for schizophrenia. Schizophr Bull 1993;19:563-577. 